\title{
Towards Chipless RFID Reading Systems Independent of Tag Orientation
}

\author{
M. Garbati, Student Member, IEEE, E. Perret, Senior Member, R. Siragusa, C. Halopè
}

\begin{abstract}
A reading technique for chipless radio frequency identification (RFID) tags is proposed. It is based on the crosspolarization principle, and unlike classical approach, the introduced technique makes it possible to read classical Chipless tag independently to their orientation. To retrieve the tag identification (ID) the property of short-time Fourier transform (STFT), and a polarization diversity approach are exploited. The former is useful to isolate the tag mode contribution from the total backscattered signal, notably the presence of clutter form surrounding objects. The latter helps to correctly excite the tag resonant scatterers and so to maximize the receiving tag mode energy. The approach has been validated with a laboratory equipment test bench solution, and with a low cost solution.
\end{abstract}

Index Terms - Chipless RFID, cross-polarization, IR-UWB radar, polarization diversity, radiofrequency.

\section{INTRODUCTION}

$\mathrm{I}^{\mathrm{r}}$ $\mathrm{N}$ chipless radio frequency identification (RFID) the tag does not have any electronic component in its design, and the RF circuit can be directly impressed with industrial printing machine [1]. To compete with barcode technology, the chipless tag needs to be read in real environments with high performance and low cost reader. To obtain such specifications, a cross-polarization reading configuration is mandatory. This configuration ensures high clutter rejection due to the scarce cross-polarization response of practical environments [4]. In a cross-polarization reading, the reader transmits a linear polarized interrogating signal towards the tag and measures the tag response in the perpendicular direction. A chipless tag working in cross-polarization needs to be aligned with the interrogating antennas to maximize the reading performances [3]. In practical application the reader must provide high read rate for automatic identification applications. It is why a cross-polarization reading approach independent of tag orientation is expected.

An orientation independent chipless tag has been introduced in [2]. Anyway, its use in practical applications is limited because the tag is not compliant with cross-polarization reading technique [3]. Till to date, no work is reported on tags which are simultaneously orientation independent and based on cross-polarization approach. Thus, a specific work on the

Marco Garbati, Etienne Perret, and Romain Siragusa are with Univ. Grenoble Alpes, Grenoble INP*, LCIS, F-26000 Valence, France. Etienne Perret is also with Institut Universitaire de France, Paris, France. Christophe Halopè is with Arjowiggins Security, Apprieu, France. (marco.garbati@lcis.grenoble-inp.fr, romain.siragusa@lcis.grenoble-inp.fr, etienne.perret@1cis.grenoble-inp.fr,Christophe.Halope@arjowiggins.com). reader part is needed. A first response was given by the authors in [3]. This paper proposes an evolution of that previous proof of concept. The introduced technique has better performances and also shows faster reading. It was experimented with a test-bench based on laboratory equipment and low cost solution, as proof of concept. The paper is organized as follow: section II describes the introducing approach and shows some simulation results. Section III presents the measurement results, and finally some conclusions are drawn.
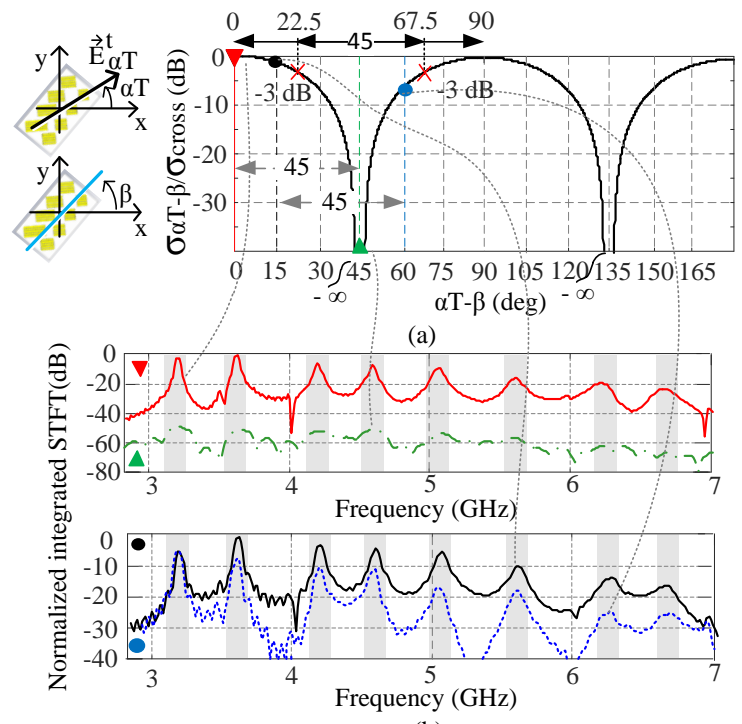

(b)

Fig. 1. (a) Variation of the tag TM-CP-RCS with tag $(\beta)$ and with the interrogating signal $\left(\alpha_{T}\right)$ direction. (b) Simulation of the tag response in frequency domain using a STFT algorithm. For each couple of measurement $\alpha_{T}$ is shifted of $45 \mathrm{deg}$.

\section{TAG ORIENTATION INDEPENDENT APPROACH}

\section{A. Theory}

In practical environment, a chipless tag response signal is composed of many components, such as the tag structural mode and the tag mode used to encode the tag identification (ID). In addition, the tag response may have contributions due to clutter, with amplitude that could be higher than the tag mode. Between all the contributions, only the tag mode has a resonant behavior, as so it is easily distinguishable using a short-time Fourier transform (STFT) [4]. In a crosspolarization based chipless RFID system, the reader is able to characterize the tag cross-polarized (CP) Radar Cross Section (RCS). The tag ID is embedded in the tag mode (TM) contribution from the CP-RCS, hereafter just noted TM-CP- 
RCS.

The variation of the TM-CP-RCS versus the angle of observation (transmitting signal direction) has been
Studio Suite 2016. The tag is composed of eight resonators; it first resonant frequency is between 3 and $7 \mathrm{GHz}$. The simulation result is shown in Fig. 1 (b). The tag ID is coded

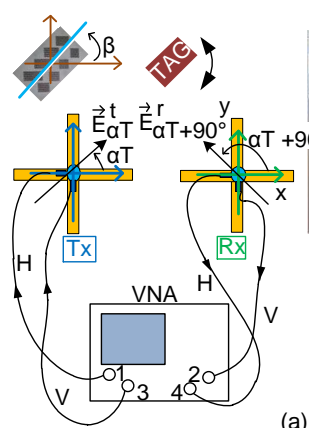

(a)

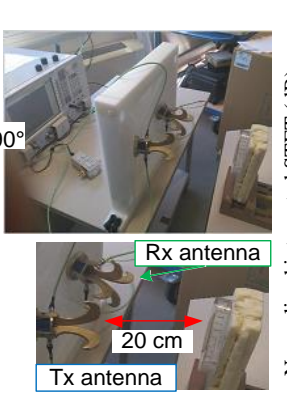

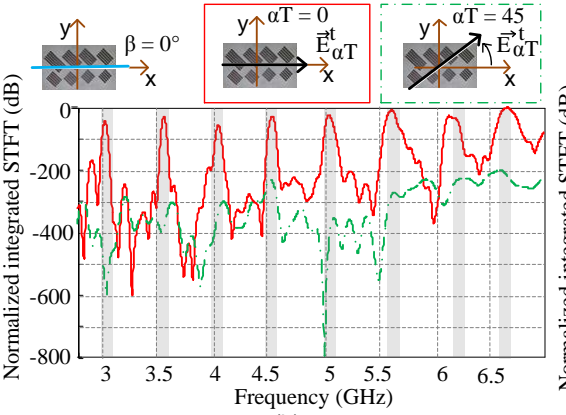

(b)

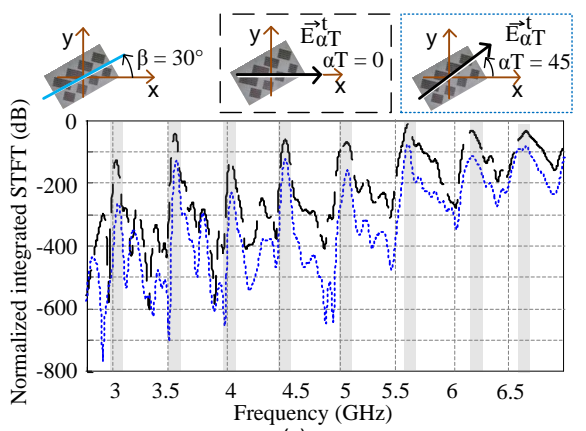

(c)

Fig. 2. (a) Reading system based on a 4 port Agilent Technologies N5222A VNA in a practical environment. The tag is placed at $20 \mathrm{~cm}$ from the closer point of the antennas. (b) Results of the proposed approach with $\beta$ equal to $0 \mathrm{deg}$, and for two values of $\alpha_{T}(0 \mathrm{deg}$ and $45 \mathrm{deg})$. (c) Results of the proposed approach with $\beta$ equal $30 \mathrm{deg}$, and $\alpha_{T}$ of 0 deg and $45 \mathrm{deg}$.

characterized in [5]:

$$
\frac{\sigma_{\alpha_{T}-\beta}}{\sigma_{\text {Cross }}}=\left|1-2 \sin \left(\alpha_{T}-\beta\right)\right|^{2}
$$

Where $\alpha_{T}$ is the angle of the reader interrogation signal, it is referred to the Cartesian coordinate system constructed on the tag plane as shown in Fig. 1 (a). $\beta$ is the angle of the tag on the same coordinate system, $\sigma_{\text {Cross }}$ is the maximum TM-CP-RCS, and $\sigma_{\alpha_{T}-\beta}$ is the actual TM-CP-RCS which depends from $\alpha_{T}-\beta$. Equation (1) is shown in semi-logarithmic scale in Fig. 1 (a), it is a periodic function of period $90 \mathrm{deg}$, and the tag shows a maximum and a minimum value $\sigma_{\alpha_{T}-\beta}$ each $90 \mathrm{deg}$. The theoretical minimum value of $\sigma_{\alpha_{T}-\beta}$ is $0(-\infty \mathrm{dB})$, and therefore the tag cannot be read in that condition. This implies a rotation of the tag, or of the incident wave to increase $\sigma_{\alpha_{T}-\beta}$. If we suppose to perform two measurements of the tag with a change of $45 \mathrm{deg}$ for $\alpha_{T}$, then at least one of the two will have an angle $\alpha_{T}-\beta$ in-between the range $\pm 22.5 \mathrm{deg}$ around one maximum of (1). This is due to the periodicity of (1) and it is shown in Fig. 1 (a). The variation of $\sigma_{\alpha_{T}-\beta}$ around $\pm 22.5 \mathrm{deg}$ of one maximum of (1) is $3 \mathrm{~dB}$ bellow $\sigma_{\alpha_{T}-\beta}$. Thus, with this technique, the tag can be read independently from its orientation.

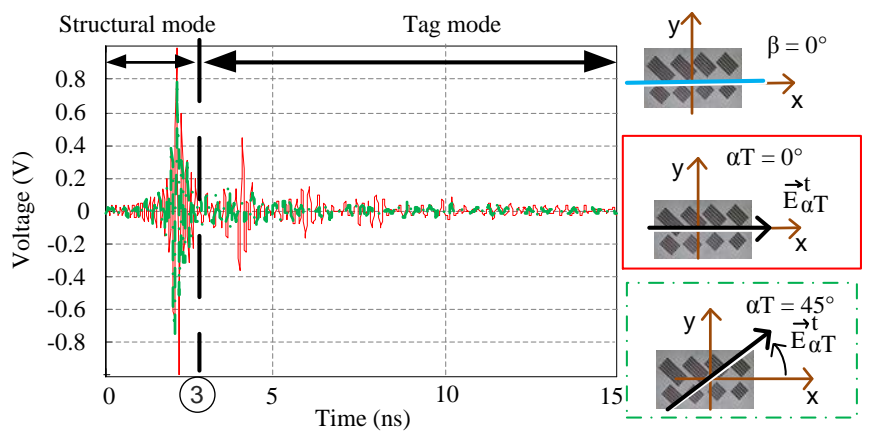

Fig. 3. Tag response in time domain corresponding to the measurement results of Fig. 2 (b) $(\beta=0)$. In red the tag response for $\alpha_{T}$ equal to $0 \mathrm{deg}$, and in dashed green the tag response for $\alpha_{T}$ equal to $45 \mathrm{deg}$.

\section{B. Simulation Result}

The cross-polarized tag used in [3,4] was simulated with CST based on the positions of the eight resonant frequencies. The tag was interrogated with a linear polarized Electro-Magnetic (EM) plane wave source, with the electric field in the direction defined by $\alpha_{T}$. The tag response was measured in crosspolarization with an electric probe. This model emulates a cross-polarized tag reading system. The tag was simulated for two value of $\alpha_{T}$ ( $0 \mathrm{deg}$ and $45 \mathrm{deg}$ ), while the tag position $\beta$ is varying in such a way to emulate the proposed reading approach. In Fig 1 (b) are shown the simulation results for $\alpha_{T}-\beta$ of 0 and $45 \mathrm{deg}$, and after for $\alpha_{T}-\beta$ of $15 \mathrm{deg}$ and $60 \mathrm{deg}$. They correspond to a tag position $\beta$ of $0 \mathrm{deg}$ and -15 deg respectively. The STFT was applied to characterize the tag mode in frequency domain that is related to the tag TMCP-RCS [4]. For real scenarios, the STFT is useful because it is a simple way to isolate the tag ID from all the disturbing parts of the backscattered signal, which can affect the reading.

To avoid a redundancy of information, in Fig. 1 (b) two representative simulation results are shown. In the first couple of measurements $\left(\alpha_{T}-\beta\right.$ of 0 and $\left.45 \mathrm{deg}\right)$ the tag ID was correctly decoded only when $\alpha_{T}-\beta$ is equal to 0 , which corresponds to one of the maximum of (1). In the other measurements $\left(\alpha_{T}-\beta\right.$ of 15 and $\left.60 \mathrm{deg}\right)$ both results show a correct reading. Anyway when $\alpha_{T}-\beta$ is equal to $15 \mathrm{deg}$, more accurate results are obtained because $\sigma_{\alpha_{T}-\beta}$ is closer to $\sigma_{\text {cross. }}$. The proposed approach has been validated by the theory and the simulation. A criterion to select which of the two measurements has to be used to decode the tag ID is given in the next section, along with some practical experimentations.

\section{EXPERIMENTAL RESULT}

The proposed approach has been tested in a practical scenario with a vector network analyzer (VNA), and then with the low cost reader proposed in [3].

The VNA was the Agilent Technologies N5222A, and the bench is shown in Fig. 2 (a). The 4 ports of the VNA were used to perform measurements with $\alpha_{T}$ equal to $0 \mathrm{deg}$, and after with $\alpha_{T}$ equal to $45 \mathrm{deg}$. The VNA ports where used in a true-mode differential measurement approach as explained in 
[3]. In transmission, the wideband dual polarized Satimo QH2000 was connected to one VNA differential logic port (physical port 1-3), and in reception another equivalent antenna was connected to the other VNA logic port (physical port 2-4). Varying the power offset of the two differential signals in transmission (logic port 1), it is possible to rotate electrically the linear polarized interrogating signal with $\alpha_{T}$ matching the selected values $(0$ and 45 deg $)$. Both measurements are in cross-polarization, therefore in reception, the receiving signal is measured at $\alpha_{T}+90 \mathrm{deg}$. For the experimentation, the tag simulated in last section was employed. It was placed at a distance of $20 \mathrm{~cm}$ from the closer point of the two antennas, and rotated in order to obtain different values of $\alpha_{T}-\beta$.

In Fig. 2 (b), the measurement results with $\beta$ equal to 0 deg (tag in horizontal position) are shown. The tag was correctly read with the first reading $\left(\alpha_{T}=0 \mathrm{deg}\right)$. It corresponds to a maximum of (1) $\left(\alpha_{T}-\beta=0 \mathrm{deg}\right)$, while the other measurement $\left(\alpha_{T}=45 \mathrm{deg}\right)$ corresponds to a minimum of (1) $\left(\alpha_{T}-\beta=45\right.$ deg). In Fig. 2 (c) the measurement results with $\beta$ equal to $30 \mathrm{deg}$ are shown. When $\alpha_{T}$ is equal to $0 \mathrm{deg}$, $\alpha_{T}-\beta$ is $-30 \mathrm{deg}$, while when $\alpha_{T}$ is equal to $45 \mathrm{deg}, \alpha_{T}-\beta$ is $15 \mathrm{deg}$. According to (1) the latter case has a higher $\sigma_{\alpha_{T}-\beta}$. From measurement results both readings were successfully, though as expected, the case $\alpha_{T}$ of 45 deg demonstrates higher accuracy. More measurements have been performed varying $\beta$, and in all cases the tag was correctly read using the proposed approach.

Note that an algorithm has to be given to select which of the two measurements must be used to retrieve the tag ID. In Fig. 3 is shown the response of the tag in time domain, with the same configuration of Fig. 2 (b). In the first $3 \mathrm{~ns}$ is present the tag structural mode, and after only the tag mode still remains. This time window corresponds to the signal part where the decoding of the information can be done. Note that the power of structural mode is higher for the case when $\alpha_{T}$ equal to 0 deg (correct reading). Anyway it is risky to use this difference as a criterion, because it can vary based on the item at which the tag is attached. A discrimination based on the tag mode is more appropriate. According to (1), the measurement that we have to take into account is the more powerful one, inside the tag mode frame.

As a low cost demonstration, the reader proposed in [3] was modified to account for measurements with $\alpha_{T}$ equal to $0 \mathrm{deg}$ and $45 \mathrm{deg}$. The reader test bench and the schematic is shown in Fig. 4(a). It makes use of the same antennas as before, with two RF switches and a splitter. The tag was placed with a $\beta$ of 45 deg that represents a case where the tag cannot be read with a classical approach ( $\alpha_{T}$ fixed at $\left.0 \mathrm{deg}\right)$. The measurement results are shown in Fig. 4 (b), in black for $\alpha_{T}$ equal to 0 deg, and in dashed red for $\alpha_{T}$ equal to $45 \mathrm{deg}$. As expected, the case with $\alpha_{T}$ equal to 45 deg shows the best reading because it maximizes (1) $\left(\alpha_{T}-\beta=0 \mathrm{deg}\right)$. All the resonance positions are correctly placed, except for the second one which presents a shifting of few tens of MHz. This imperfection is most due to the sensibility of the reader which is of $-60 \mathrm{~dB}$. In case of
$\alpha_{T}$ equal to 0 deg only the first resonance is at the correct position.

The experimental results demonstrate that this technique can be used to read the tag whatever its orientation, maintaining a cross-polarization approach. The limitation of this method is the use of two dual polarized antennas, such as the Satimo QH2000, instead of one as for the classical approach. The transmitting and receiving antennas are less lined up with the tag. Thus, the receiving signal is attenuated of few $\mathrm{dB}$ depending on antennas, and tag directivity. It also represents an additional reader realization cost. The use of only one antenna is feasible using a circulator for each antenna port. It will compensate for the lower directivity, but will increase the reader leakage caused by the circulators.

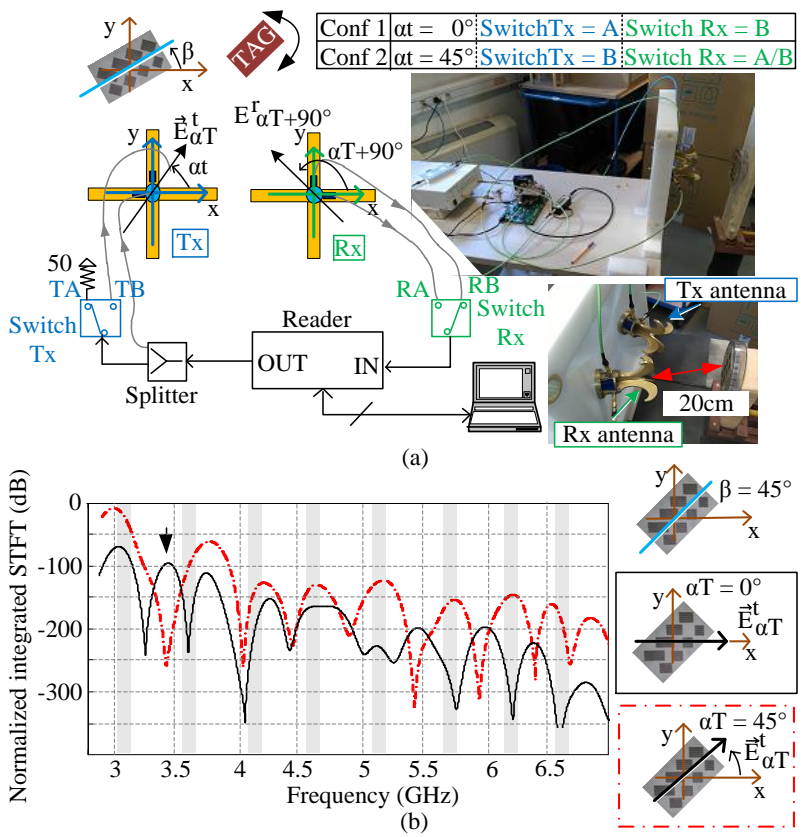

Fig. 4. (a) Bench of the low cost reader used to read the tag with $\beta$ equal to $45 \mathrm{deg}$ using the proposed approach ( $\alpha_{T}$ equal to $0 \mathrm{deg}$ and $45 \mathrm{deg}$ ). (b) Measurement results. Better results are shown for the case with $\alpha_{T}$ equal to $45 \mathrm{deg}$ that match the positions of the tag resonant frequencies.

\section{CONCLUSION}

This paper has presented a chipless RFID reading method, based on cross-polarization approach, which does not need an alignment between the tag and the reader. The reader performs measurements in two discrete directions, which are enough to correctly retrieve the tag ID. The STFT algorithm is used to remove the tag structural mode contribution and clutter. In the demonstrated implementations, the reader was equipped with two dual polarized antennas. This increases the reader cost, and may reduce the tag reading range depending on the antennas and tag directivity. However, regarding the tag orientation, this method does not give any constraint to the tag design.

\section{REFERENCES}

[1] Munawar M. Khan, Farooq A. Tahir, M. F. Farooqui, Atif Shamim, Hammad M. Cheema, "3.56 bits/cm2 Compact Inkjet Printed and Application Specific Chipless RFID Tag", IEEE Antenna and Wireless Propag. Lett., Vol. 15, pp. 1109-1112, 2015 
[2] M. A. Islam, Y. Yap, N. Karmakar, and A. K. M. Azad, "Orientation Independent Compact Chipless RFID Tag," in Proc. IEEE 2012 Int. Conf. on RFID - Techn. and Appl., Nice, FR, 2012, pp. 137-141.

[3] M. Garbati, A. Ramos, R. Siragusa, E. Perret, and C. Halopé, "Chipless RFID reading system independent of polarization," Proc. IEEE MTT-S Int. Microw. Symp. (IMS), San Francisco, CA, 2016, pp. 1-3.

[4] A. Ramos, E. Perret, O. Rance, S. Tedjini, A. Lázaro, and D. Girbau, "Temporal Separation Detection for Chipless Depolarizing FrequencyCoded RFID," in IEEE Trans. Microw. Theory Techn., vol. 64, no. 7, pp. 2326-2337, July 2016.

[5] O. Rance, R. Siragusa, P. Lemaître-Auger and E. Perret, "Magnitude Coding Technique," in RCS Synthesis for Chipless RFID: Theory and Design, 1th ed., ISTE Press - Elsevier, 2017. 